\title{
Computer Games: A Double-Edged Sword?
}

\author{
De-Lin Sun, B.S., Ning Ma, B.S., Min Bao, Ph.D., Xiang-Chuan Chen, Ph.D., and Da-Ren Zhang
}

\begin{abstract}
Excessive computer game playing (ECGP) has already become a serious social problem. However, limited data from experimental lab studies are available about the negative consequences of ECGP on players' cognitive characteristics. In the present study, we compared three groups of participants (current ECGP participants, previous ECGP participants, and control participants) on a Multiple Object Tracking (MOT) task. The previous ECGP participants performed significantly better than the control participants, which suggested a facilitation effect of computer games on visuospatial abilities. Moreover, the current ECGP participants performed significantly worse than the previous ECGP participants. This more important finding indicates that ECGP may be related to cognitive deficits. Implications of this study are discussed.
\end{abstract}

\section{Introduction}

$\mathrm{C}$ COMPUTER GAMES have become a part of our everyday lives. Players use games to gain powers, to compete or cooperate with others, or just to escape their real-life problems. ${ }^{1}$ Today, in China alone, there are about 40 million people enjoying online games ${ }^{2}$ (a subtype of computer games). With this soaring number of game players, ECGP has already become a serious social problem, ${ }^{3-5}$ sometimes called "heroinware" 6 or "game addiction." 7 Many players are so involved in games that they risk losing significant relationships, jobs, and education or career opportunities. ${ }^{3-5}$ However, experimental lab studies on the negative consequences of ECGP on players' cognitive abilities are still scarce, and little is known about the ECGP participants' cognitive characteristics.

Previous surveys using questionnaires showed that ECGP is related to negative emotions, loss of control, $, 5,7$ and even inattention and ADHD. ${ }^{8}$ Therefore, in performing some cognitive tasks, participants who play games excessively may encounter many internal (e.g., poor emotional state) or external (e.g., interference from task-unrelated stimuli) disturbances. These may lead to their tasks' failure. However, the effects of game playing are complicated. Some research shows that computer-game training can improve a range of visuoperceptual and attentional skills. ${ }^{9-13}$ Therefore, players who spend excessive time playing games may suffer the negative consequences of ECGP and simultaneously benefit from the positive effects of game training, making it difficult to measure the positive and the negative consequences clearly.

However, the positive effects of game playing may last longer than the negative ones after players reduce playing time. Adverse effects, as Griffiths ${ }^{14}$ says, tend to be temporary and are resolved spontaneously with decreased frequency of play. When a player reduces playing time, his or her ECGP score, an index of the negative consequences of excessive playing, ${ }^{5}$ also drops. On the contrary, a previous study showed that some types of perceptual experience lead to long-term neural changes (detectable 2 or more years after the experience)..$^{15}$ Moreover, computer games, especially those that require players to distribute and switch attention quickly around the field, can alter a range of visual skills. ${ }^{9}$ Therefore, computer game players may benefit from their games training for some time even without further experience.

In the present study, we compared the players who still play games excessively with those who no longer practice ECGP in order to examine the negative effects of ECGP more clearly.

The multiple object tracking (MOT) task has been used to show the positive effects of game playing. ${ }^{10}$ This task requires participants to track positions of a number of target items for a few seconds during which the participant is vulnerable to disturbances. ${ }^{16}$ Therefore, we hypothesized that different groups of participants would show dissimilar performance of the MOT task.

Hefei National Laboratory for Physical Sciences, Microscale and School of Life Sciences, University of Science \& Technology of China, Hefei, Anhui, China. 


\section{Methods}

\section{Participants}

Sixty-five male graduate or undergraduate students were recruited from the University of Science and Technology of China (USTC). Only male participants were selected because gender effect was not a focus of this study and because of the scarcity of female ECGP participants. All participants gave their informed consents before the experiments and received monetary compensation for their time. None of the participants reported a history of drug abuse or head injury. Participants' most favorite game types included intense realtime strategy games (e.g., StarCraft, WarCraft III), first-person shooting games (e.g., Counter-Strike, Quake III), and massive multiplayer online games (e.g., World of Warcraft). All these games require players to distribute and switch attention quickly around the field, which is the most prominent characteristic of action video games. ${ }^{9}$ One of the participants was excluded for colorblindness. Another two players were excluded because their favorite game was Pro Evolution Soccer, which requires players to track targets moving onscreen and is much more like the MOT task than other games. Therefore, it may provide extra training on cognitive abilities related to MOT tasks. In addition, two participants were excluded for incomplete data. Thus, 60 participants were included in the final analysis.

\section{ECGP scores and playing time}

All participants' ECGP scores were evaluated prior to the experiment via the problem videogame-playing questionnaire, ${ }^{5}$ a 9-item questionnaire adapted from DSM-IV for substance abuse and pathological gambling. ${ }^{17}$ which we translated into Chinese. The questionnaire addresses issues such as being preoccupied by playing, loss of control, negative feelings when not playing, and poor physical or psychological consequences. Participants were instructed to give a yes or no answer to each question. Higher score indicates more negative effects caused by ECGP. Two types of ECGP score were obtained for each participant: the highest score (HES) during the past year before the experiment and the current score (CES).

Based on their HES and CES, participants were divided into three groups of 20 (Figure 1A): the current ECGP group (cECGP: HES $>5, \mathrm{CES}>5$ ), the previous ECGP group (pECGP: HES $>5$, CES $<=5$ ), and the control group (control: $\mathrm{HES}<=5, \mathrm{CES}<=5$ ). The mean ages were $20.7 \pm$ 1.4 (cECGP), $21.5 \pm 2.4$ (pECGP), and $19.6 \pm 1.1$ (control).

Forty-eight participants (16 in cECGP group, 13 in pECGP group, and 19 in control group) reported the hours spent on computer games per week when they had the HES $(\mathrm{HH})$ as well as when they participated this experiment $(\mathrm{CH})$ (Figure 1B).

\section{MOT task}

The stimuli used in the MOT task were 12 bouncing balls (each $1.6^{\circ}$ in diameter) moving independently at a constant velocity $\left(5.3^{\circ} / \mathrm{s}\right)$ within a square frame $\left(20.4^{\circ} \times 20.4^{\circ}\right)$ on a black background. The balls repelled each other and bounced off the edge of the frame. All the balls moved smoothly with no sudden transition (Figure 1C).

A MOT task trial started with a visual cue (a white word "Tracking," $4.8^{\circ} \times 3.2^{\circ}$ ) presented in the center of the square frame for $1 \mathrm{sec}$. Following the cue, 12 green balls appeared and moved in a Brownian-like pattern. After $1 \mathrm{sec}$, a variable number $(2,4$, or 6$)$ of the balls (targets) turned red for $2 \mathrm{sec}$ and then turned back. This subset of balls was tracked by the participants for $13 \mathrm{sec}$. After that, all the balls stopped moving, and the participants were required to point out the targets by clicking the left mouse button on them one by one. When the number of balls clicked reached the target number in that trial, the next trial would begin. A trial was considered to be completed correctly only when all the targets in the trial were reported.

There were three tracking conditions: to track 2, 4, or 6 targets. Each condition had 3 blocks, consisting of 9 trials each for a total 81 trials (27 trials per condition). There was a 2min rest between each block. The order of the trials was pseudorandomized through the experiment. Before the formal trials, all participants performed two practice trials (a 2target trial and a 6-target trial, each different from the formal trials).

\section{Results}

The mean accuracy of the MOT task in three groups is shown in Figure 1D. A repeated measure ANOVA $(3 \times 3)$ was used to analyze these data, with group (cECGP, pECGP, and control) as a between-participant factor and load $(2,4$, and 6 targets) as a within-participant factor. The results showed significant main effects of group $(F(2,57)=7.7, p=$ $0.001)$ and load $(F(1.5,87.2)=459.9, p<0.001$, GreenhouseGeiser corrected), as well as significant interaction of group by load $(F(3.1,87.2)=6.9, p<0.001$, Greenhouse-Geiser corrected). Furthermore, in the 6-target balls condition, paired comparisons (post hoc analysis, Bonferroni corrected) between the groups showed that the pECGP group had significantly higher accuracy than both the cECGP $(p<0.001)$ and the control groups $(p=0.019)$, and there was no significant difference between the latter two groups $(p=0.460)$. Paired comparisons were also used to compare ECGP scores (HES and CES) and playing time $(\mathrm{HH}$ and $\mathrm{CH}$ ) between groups (see Figure $1 \mathrm{~A}$ and $\mathrm{B}$ ).

\section{Discussion}

Participants who had previously trained but were not currently active in gaming (pECGP group) and were disturbed little by ECGP at the time of experiment performed significantly better than the control participants, showing the persistent positive effects of gaming. Moreover, and more importantly, compared with the pECGP participants, poor performance of the cECGP participants showed significantly negative consequences of excessive playing on their cognitive abilities. This result was consistent with a previous survey in which attention deficits in gamers were reported by their parents ${ }^{8}$ and provided more direct and objective evidence.

Therefore, for cECGP participants, game playing presents both positive and negative consequences at the same time. This point was supported by our result that no significant differences in MOT task performance were found between the cECGP participants and the controls in the present study. The negative effects related to ECGP may be offset by the positive consequences of game playing. It may provide a potential reason that so few lab experimental studies have re- 

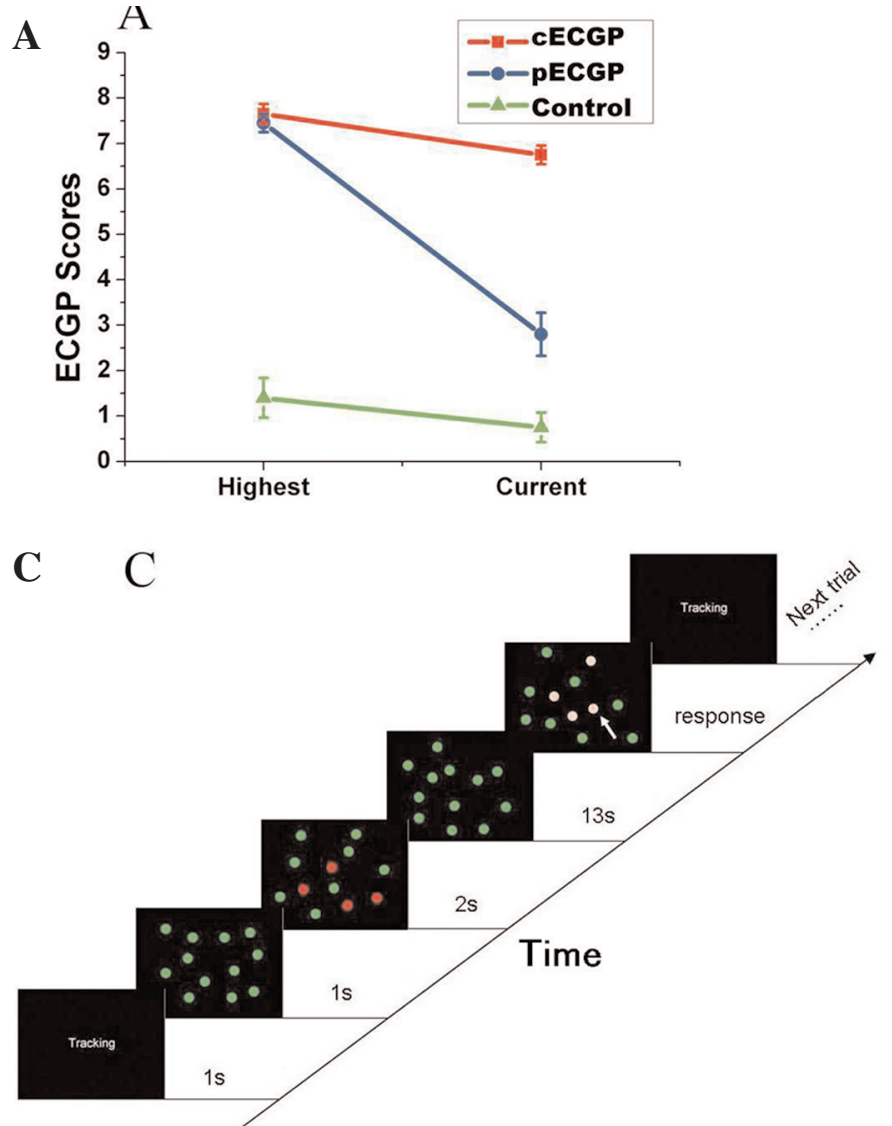

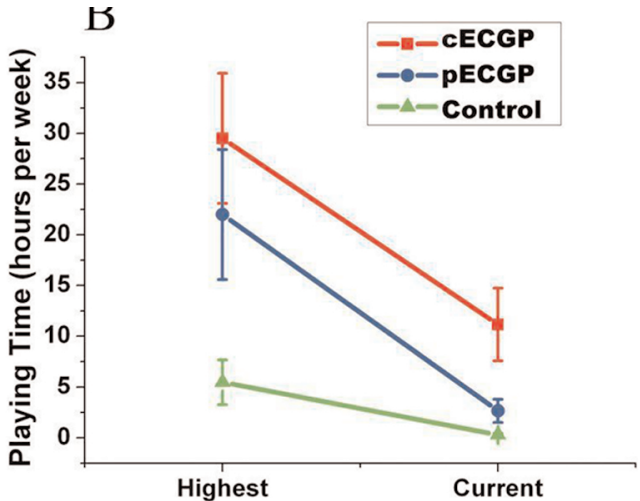

B

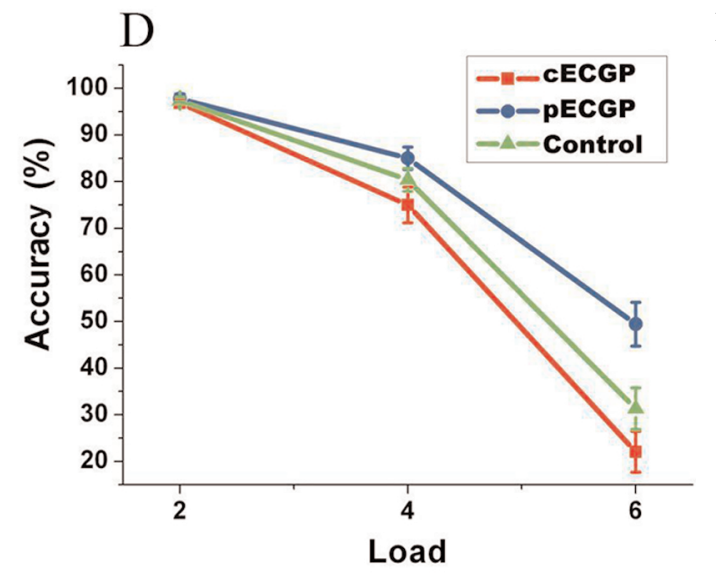

D

FIG. 1. (A) Excessive computer game playing scores (HES and CES). The HES of the cECGP and the pECGP participants were higher than those of the control participants $(p<0.001)$, while there was no significant difference between the former two groups $(p=1.000)$. The CES of the cECGP participants were higher than those of the pECGP participants $(p<0.001)$, while the CES of the pECGP participants were higher than those of the control group participants $(p<0.001)$. (B) Hours per week spent on computer game playing $(\mathrm{HH}$ and $\mathrm{CH})$. cECGP participants' $\mathrm{HH}$ were significantly longer than those of the control group participants $(p=0.003)$, and the pECGP participants' HH were marginally longer than those of the control participants $(p=0.079)$. However, no significant difference in HH was found between the cECGP group and the pECGP group $(p=0.960)$. $\mathrm{CH}$ of the cECGP group was much longer than that of the pECGP group $(p=0.032)$ and the control group $(p=0.002)$, and no significant difference was found between the latter two groups $(p=1.000)$. (C) Schematic depiction of the MOT task (a 4-targets trial is shown as an example). When all balls stopped, the participants were required to point out the targets (pink balls here but green balls in real experiment) by clicking the left mouse button on them (white arrow here, which indicates the mouse pointer). (D) MOT performance of three groups at different load levels. In 6-targets condition, the pECGP participants performed significantly better than both the control participants $(p=0.019)$ and the cECGP participants $(p<0.001)$, and no significant difference was found between the latter two groups $(p=0.460)$. All $p$ values are from paired comparisons between groups (post hoc analysis) and were corrected by Bonferroni correction. Error bars denote the standard error of the mean. (Color version of figure can be viewed online at www.liebertonline.com)

ported cognitive declines related to ECGP. Therefore, the current study not only proved the existence of cognitive deficits caused by game playing but also provided a new way to study these negative effects.

As far as we know, few previous studies show that cultural or socioeconomic factors directly influence the ability to process information about several objects simultaneously-a basic human ability. Therefore, it seems that the current results should be generalized. However, previous findings showed that the MOT task performance was affected by age $\mathrm{e}^{18,19}$ and types of games (i.e., players experienced in action games performed better than those experienced in nonaction games; see Green and Bavelier ${ }^{10}$ ). Therefore, generalizing the current results by age group or game types should be done cautiously. A recent survey ${ }^{20}$ on online games showed that most Chinese players are under 30 years old and prefer action or actionlike games (games that require players to distribute attention quickly around the field; see Green and Bavelier ${ }^{9}$ ). A surprising study ${ }^{21}$ from America Online (AOL) found that females over 40 spent more time playing online games than did other measured groups, and they often stayed up late at night with word and puzzle challenges. Because of the differences in age and preferred games, American cECGP participants and pECGP participants may perform differently from their Chinese counterparts. Therefore, an interesting topic about culture's influence on basic cognitive functions should be further studied in the future. 
An important and open question is why some players can break away from excessive playing (e.g., the participants in the pECGP group) while many others continue to indulge in computer games. The investigation of the neural basis of ECGP and the effects of gender, age and genetics on players' behaviors may shed light on this question.

\section{Acknowledgments}

This research is supported by the National Nature Science Foundation of China (30370478, 30470572, 30670683, and 30770713) and the Ministry of Science and Technology of China (2006CB500705). We thank Dr. Zhao-Xin Wang, XiaoChu Zhang, Peng Zhang, Xiang Wu, and Bin Xuan for their advice on this paper.

\section{Disclosure Statement}

The authors have no conflict of interest.

\section{References}

1. Yee N. Motivations for play in online games. Cyberpsychology \& Behavior 2006; 9:772-5.

2. China Internet Network Information Center. Semiyearly report 2007: the 20th survey of the development of Chinese Internet network. www.cnnic.net.cn/uploadfiles/pdf/2007/ 7/18/113918.pdf (accessed July 2007).

3. Young KS. Internet addiction: the emergence of a new clinical disorder. Cyberpsychology \& Behavior 1998; 1:237-44.

4. Allison SE, von Wahlde L, Shockley T, et al. The development of the self in the era of the Internet and role-playing fantasy games. American Journal of Psychiatry 2006; 163: 381-5.

5. Tejeiro Salguero RA, Moran RM. Measuring problem video game playing in adolescents. Addiction 2002; 97:1601-6.

6. Ng BD, Wiemer-Hastings P. Addiction to the Internet and online gaming. Cyberpsychology \& Behavior 2005; 8:110-3.

7. Grusser SM, Thalemann R, Griffiths MD. Excessive computer game playing: evidence for addiction and aggression? Cyberpsychology \& Behavior 2007; 10:290-2.

8. Chan PA, Rabinowitz T. A cross-sectional analysis of video games and attention deficit hyperactivity disorder symptoms in adolescents. Annals of General Psychiatry 2006; 5:16.
9. Green CS, Bavelier D. Action video game modifies visual selective attention. Nature 2003; 423:534-7.

10. Green CS, Bavelier D. Enumeration versus multiple object tracking: the case of action video game players. Cognition 2006; 101:217-45.

11. Green CS, Bavelier D. Effect of action video games on the spatial distribution of visuospatial attention. Journal of Experimental Psychology: Human Perception \& Performance 2006; 32:1465-78.

12. Green CS, Bavelier D. Action-video-game experience alters the spatial resolution of vision. Psychological Science 2007; 18:88-94.

13. Miller G. Society for neuroscience meeting. Computer game sharpens aging minds. Science 2005; 310:1261.

14. Griffiths M. Video games and health. British Medical Journal 2005; 331:122-3.

15. Karni A, Sagi D. The time course of learning a visual skill. Nature 1993; 365:250-2.

16. Pylyshyn ZW. Some puzzling findings in multiple object tracking (MOT): II. Inhibition of moving nontargets. Visual Cognition 2006; 14:175-98.

17. American Psychiatric Association (APA). (1994). Diagnostic and statistical manual of mental disorders, 4th ed. Washington, DC: Author.

18. Trick LM. Multiple-object tracking in children: the "Catch the Spies" task. Cognitive Development 2005; 20:373-87.

19. Trick LM, Per T, Sethi N. Age-related differences in multiple-object tracking. Journal of Gerontology: Psychological Sciences 2005; 60:102-5.

20. iResearch Consulting Group. (2007) Yearly report 2006. The 6th Chinese Online Game Research Report. http://china. 17173.com/2006/dcbg.doc (accessed May 2007).

21. Greenspan R. (2004) Girl gamers grow up. www.clickz.com/ 3312301/print (accessed July 2008).

Address reprint requests to: Da-Ren Zhang

Hefei National Laboratory for Physical Sciences Microscale and School of Life Sciences University of Science \& Technology of China Hefei, Anhui 230027

China

E-mail: drzhang@ustc.edu.cn 